\title{
AgNORs in cardiomyocytes from surgical patients with coronary heart disease
}

N N Mamaev, O V Kovalyeva, Kh K Amineva, A Ya Gudkova, Yu B Maier, I S Polykarpov, Yu A Schneider, S N Proshin, L V Lebedev

\begin{abstract}
Aim-To evaluate the interphase ribosomal RNA cistron activity of cardiomyocytes in surgical patients with chronic ischaemic heart disease by means of the nucleolar organiser region silver staining (AgNOR) technique.

Methods-Nucleoli were investigated in myocardial samples obtained from 46 patients with chronic ischaemic heart disease before, during, and soon after cardioplegia ischaemia. Cryostat sections of $10 \mu \mathrm{m}$ thickness were air dried, fixed in methanol/glacial acetic acid (3:1) for 15 minutes, rinsed carefully with distilled water, incubated in $2 \mathrm{~N}$ formic acid for 10 minutes, and impregnated with silver colloid solution for $2.5-3$ minutes at $68-70^{\circ} \mathrm{C}$. The lightly counterstained sections were examined under oil immersion at $\times 1000$ magnification. For the estimation of AgNOR numbers at least 100 silver stained cardiomyocyte and fibroblast nuclei were counted in each section. On the basis of these data, the mean number of AgNORs in each nucleus was determined. The Student's $t$ test was used to compare the groups tested.
\end{abstract}

Division of Cardiology, Department of Internal Medicine I, Pavlov Medical University of $\mathbf{S t}$ Petersburg, Leo Tolstoi Str 6/8, St Petersburg 197089, Russia N N Mamaev

O V Kovalyeva

A Ya Gudkova

Yu B Maier

S N Proshin

Division of

Cardiosurgery,

Department of

Surgery II, Pavlov

Medical University of

St Petersburg

I S Polykarpov

Yu A Schneider

L V Lebedev

Department of Pathology, Pavlov Medical University of St Petersburg

Kh K Amineva

Correspondence to: Dr Mamaev.

Accepted for publication 28 May 1998
Results-The initial mean numbers of AgNORs varied greatly, demonstrating a difference between groups of patients with or without antecedent myocardial infarction $(9.5 v 11.0 ; p<0.05)$. During myocardial arrest, the numbers of AgNORs in cardiomyocytes were decreased in all but seven patients, while those in fibroblasts tended to increase. At the stage of reperfusion and myocardial warming, in all but three patients the numbers of AgNORs in cardiomyocytes either normalised or were even higher than the initial value.

Conclusions-The AgNOR count in cardiomyocytes is a very sensitive test for the measurement of cardiac function in surgical patients with chronic ischaemic heart disease and could be useful for monitoring myocardial status during the course of surgery, including cardioplegia. The high risk group for surgery included patients with antecedent myocardial infarction and severe heart failure. It is thought that a reversible nucleolin/fibrillarin/prerRNA/small nucleolar RNA modification might account for this fast decline then rise in the AgNOR count in cardiomyocytes at the stages of cardioplegia and reperfusion, respectively.

(F Clin Pathol: Mol Pathol 1998;51:218-221)
Keywords: coronary heart disease; cardioplegia; nucleolar organiser regions; pre-rRNA synthesis/ processing

Recently, it has been shown that the mean numbers of interphase silver stained nuclear organiser regions (AgNORs) in cardiomyocytes from patients with chronic ischaemic heart disease $\mathrm{e}^{12}$ decline in keeping with a reduced expression of mRNAs ${ }^{3}$ and a deficiency of many myocardial proteins. ${ }^{4-6}$ Our first results concerning cardiomyocyte AgNORs were obtained in postmortem myocardial samples $^{2}$ and here we present our recent findings of changes in AgNOR numbers in myocardial samples taken from chronic ischaemic heart disease patients operated upon under the conditions of cold chemical cardioplegia. It is known that the cardioprotective strategies available for intraoperative management are numerous $^{78}$; also there is no clear evidence yet that one method is ideal. The release of cardiotropin I is a suitable marker of myocardial injury at the cardioplegia stage. ${ }^{9}$ However, increased cardiotropin I release is related to significant myocardial damage, which seldom occurs in well protected myocardium. The assessment of interphase AgNORs in cardiomyocytes is well suited to the investigation of these specimens. ${ }^{121011}$ Furthermore, myocardial fibroblasts can be studied in a similar way. Our paper presents some new data obtained from myocardial samples taken before and at the stages of both cardioplegia and reperfusion from 46 surgical patients with chronic ischaemic heart disease.

Patients and methods

The study was carried out on myocardial samples obtained from 46 patients with chronic ischaemic heart disease ( 44 men, two women; mean age, 46.4 years) who were operated upon under the conditions of cold chemical cardioplegia at the cardiac surgery department of the Pavlov Medical Institute in the period between 1986 and 1988. Small pieces of the left ventricular free wall $(n=35)$ or the right antrum $(n=11)$ were obtained serially before severe myocardial ischaemia (46 patients) and at subsequent reperfusion (22 patients). The duration of the disease ranged from four months to 17 years (mean, 6.2 years). Twenty two of 46 patients had angina pectoris of functional classes II or III. Nineteen patients had a previous history of myocardial infarction, which was complicated by left ventricular aneurysm in six cases. Thirty four of 46 patients had previous systemic hypertension. 
Table 1 Clinical details and the results of cardiomyocyte nucleolar silver staining from surgical patients with coronary heart disease

\begin{tabular}{|c|c|c|c|c|c|c|c|c|}
\hline \multirow[b]{3}{*}{ Group } & \multirow[b]{3}{*}{ (No.) } & \multirow[b]{3}{*}{$M I$ (no.) } & \multirow[b]{3}{*}{$H F(n o)}$. & \multirow[b]{3}{*}{ AN duration (minutes) } & \multirow[b]{3}{*}{ AC duration (minutes) } & \multicolumn{3}{|c|}{ Number of AgNORs } \\
\hline & & & & & & \multicolumn{3}{|c|}{ Operation stages } \\
\hline & & & & & & $A$ & $B$ & $C$ \\
\hline $\begin{array}{l}\text { Control } \\
\text { CHD }\end{array}$ & 5 & 0 & 0 & $27.0(0.7)$ & $48.2(3.6)$ & $7.6(0.9)$ & $6.9(1.0)$ & $9.1(0.5)$ \\
\hline Left ventricle & 36 & $29^{\star}$ & 17 & $39.0(4.5)$ & $77.5(4.9)$ & $10.1(0.36)$ & $9.8(0.5)$ & $13.0(0.6)$ \\
\hline Right antrum & 11 & $14^{\star}$ & 11 & $38.0(3.5)$ & $80.0(4.8)$ & $9.8(0.5)$ & $10.0(2.2)$ & $10.7(0.6)$ \\
\hline
\end{tabular}

Values are mean (SEM)

*Indicates that the numbers of antecedent MIs in some patients exceeded one.

A, the stage of operation before anoxia; B, global ischaemia and cold chemical cardioplegia; $\mathrm{C}$, reperfusion and myocardial rewarming stage; AC, artificial circulation; $\mathrm{AN}$, anoxia; CHD, coronary heart disease; HF, heart failure; MI, myocardial infarction.

Moreover, half of the patients had clinical signs of heart failure that was classified (according to New York Heart Association; NYHA) as NYHA class II or III. The operation was carried out under hypothermic perfusion (18$\left.20^{\circ} \mathrm{C}\right)$. The mean (SEM) duration of artificial circulation was 80 (4.8) minutes. The duration of aorta clamping ranged from 20 to $105 \mathrm{~min}$ utes (mean, 38.1 minutes; SEM, 3.52). Cold potassium cardioplegia, sometimes with blood, was used. Also, myocardial protection was enhanced by additional external topical cooling by means of antiseptic physiological saline solution cooled to $1^{\circ} \mathrm{C}$. The myocardial samples obtained during surgery were put into liquid nitrogen. Cryostat sections of $10 \mu \mathrm{m}$ thickness were air dried, fixed in methanol/ acetic glacial acid mixture (3:1) for 15 minutes, and rinsed thoroughly with distilled or ionised water. After repeated air drying, the sections were put into $2 \mathrm{~N}$ formic acid for 10 minutes, rinsed again, and impregnated with a mixture of $50 \%$ aqueous solution of silver nitrate with gelatin at $68-70^{\circ} \mathrm{C}$, according to the method of Howell and Black, ${ }^{12}$ with a slight modification. Two drops of gelatin were placed on the slide and allowed to mix with four drops of freshly prepared aqueous AgNOR solution (50\%). The slides were covered by cover glasses, placed on to filter paper in a wet chamber (Petri dish), and incubated at $68-70^{\circ} \mathrm{C}$ for $2.5-3$ minutes. The cover glasses were removed and the slides were washed carefully with distilled
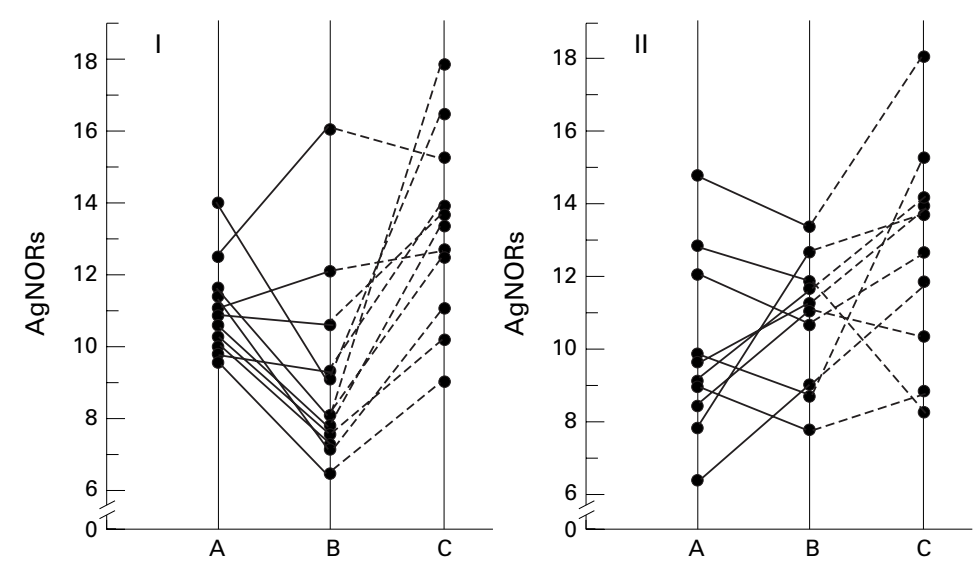

Figure 1 Serial assessment of AgNOR numbers in cardiomyocyte nucleoli from surgical patients with a stable (I) or progressive (II) clinical course of coronary heart disease. $A, B$, and $C$ are findings before anoxia; with ischaemia and cold chemical cardioplegia; and at the stage of reperfusion and myocardial rewarming, respectively. A significant increase of AgNOR numbers during global myocardial ischaemia and cold chemical cardioplegia compared with initial findings indicates an inadequate myocardial protection and vice versa. water. Then the slides were counterstained in $2 \%$ Giemsa/phosphate buffer solution (pH 6.8) for 10 seconds, rinsed three times in distilled water, air dried, and mounted. The numbers of individual AgNORs in each cell were counted under oil immersion at $\times 1000$ magnification. At least 100 silver stained cardiomyocyte or fibroblast nuclei were examined. On the basis of these data the mean numbers of AgNORs in each nucleus were calculated. The initial data from five surgical patients with ventricular septal defect were used as controls. Comparison of mean values was performed by means of the Student's $t$ test. All results are expressed as the mean (SEM).

\section{Results}

Some clinical details and the results of cardiomyocyte and fibroblast silver staining are presented in table 1 and figs $1-3$. In both controls and the patients with chronic ischaemic heart disease the initial numbers of AgNORs varied greatly (table 1), being higher in patients with chronic ischaemic heart disease (7.6 v $10.1 ; \mathrm{p}<0.01$ ), which could be explained by compensative hypertrophy of cardiomyocytes in the myocardium of patients with chronic ischaemic heart disease. The mean numbers of AgNORs in chronic ischaemic heart disease samples taken from the left ventricle and the right antrum were similar, although their numbers differed in patients with antecedent myocardial infarction. In the latter cases, the AgNOR numbers in ventricular cardiomyocytes diminished and those in the right antrum cardiomyocytes increased. Also, there was an inverse correlation between the decrease in AgNORs and chronic ischaemic heart disease duration and severity. Finally, the mean numbers of AgNORs were smaller in patients with antecedent myocardial infarction and left ventricular aneurysm $(\mathrm{p}<0.05)$.

The cardiomyocyte response to global ischaemia and to cold chemical cardioplegia varied (fig 1). In all but nine patients, the mean numbers of AgNORs were decreased. However, this trend was weak in two patients and even inversed in seven others. Among the latter were two patients with a relatively benign clinical course of chronic ischaemic heart disease (angina pectoris of functional class III) and five patients with a severe clinical course of chronic ischaemic heart disease (angina pectoris of functional class IV, antecedent myocardial infarctions complicated by left ventricular aneurysm and severe heart failure). As for the 


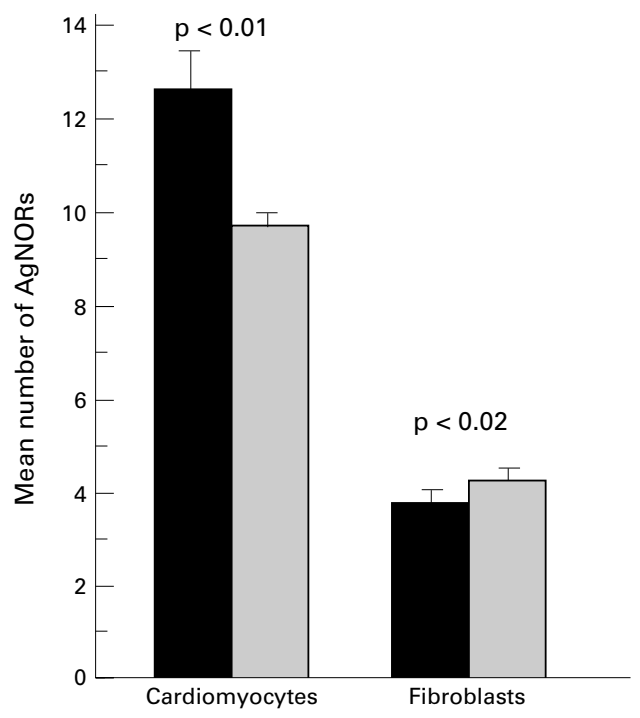

Figure 2 Differences in AgNOR numbers before anoxia (black bars) and during cold chemical cardioplegia (grey bars) in cardiomyocytes and fibroblasts from surgical patients with coronary heart disease.

AgNOR numbers in fibroblasts, there was a tendency to increase under cardioplegia in most patients (fig 2). Simultaneous investigation of succinate dehydrogenase activity showed that it declined significantly in cardiomyocytes from patients with inadequate myocardial protection (fig 3B) and that this did not occur in the group of patients with a natural AgNOR decrease with global ischaemia and cold chemical cardioplegia (fig 3A).

At the stage of reperfusion and myocardial warming, the AgNOR numbers in cardiomyocytes were mostly higher than at the previous stage (fig 1).

Additional analysis of the data showed that the mean numbers of AgNORs in cardiomyocytes at the reperfusion stage were increasing (compared with the initial value), and were much higher in patients with chronic ischaemic heart disease without previous myocardial infarctions than in those with myocardial infarctions (11.2 and $14.6 v 9.6$ and 11.8, respec-
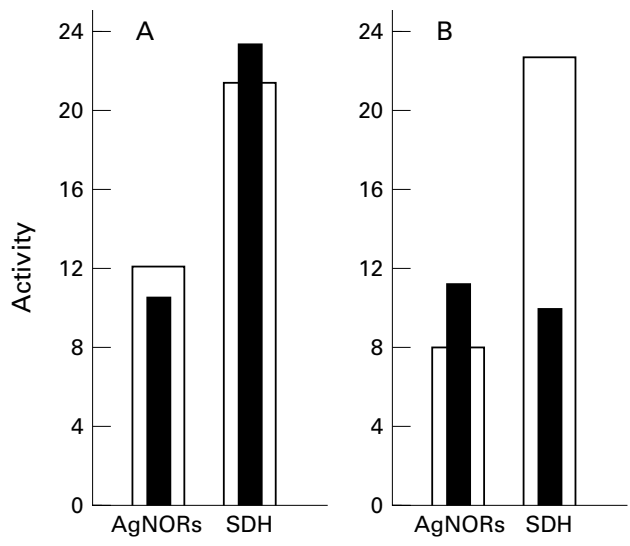

Figure 3 The activity of nucleolar organiser regions (AgNOR numbers) and succinate dehydrogenase (SHD, arbitrary units) in cardiomyocytes from patients with $(A)$ an effective or (B) non-effective myocardial protection in relation to global ischaemia. White and black columns represent the results before anoxia and with global ischaemia, respectively. tively; $\mathrm{p}>0.05)$. Also, cardiomyocyte AgNOR numbers increased to a greater extent in patients without pre-operative heart failure compared with those with this complication (10.5 and $13.7 v 11.3$ and 13.0, respectively). Finally, according to our data, the response of the cardiomyocyte protein synthesising machine was related to the presence or absence of left ventricular aneurysm.

\section{Discussion}

Our study shows that the numbers of AgNORs in cardiomyocytes from patients with chronic ischaemic heart disease varies greatly, being minimal in cases with a previous history of myocardial infarction. At the stage of total ischaemia and cold chemical cardioplegia the initial AgNOR number decreased in all but seven patients. The group with inadequate myocardial protection included patients with antecedent myocardial infarction(s) coupled with left ventricular aneurysm and heart failure in two cases. The AgNOR numbers in cardiomyocytes at the stage of reperfusion and myocardial warming compared to anoxia increased in all but three patients, being mostly higher than in the initial sample. In our opinion, the last phenomenon might be ascribed to changes in the protein synthesising mechanism of cardiomyocytes in relation to damage at the stage of global ischaemia and cardioplegia. This assumption is supported in part by our finding that pre-rRNA synthesis/processing is less active at the stage of reperfusion in cardiomyocytes from patients with antecedent myocardial infarction. Thus, the main cytological finding of our study was that a rapid decline in AgNOR numbers in cardiomyocytes at the stage of total myocardial ischaemia and cold chemical cardioplegia is followed by a fast rise in the count at the stage of reperfusion and myocardial rewarming. It has been shown recently in postmortem myocardial samples that heart failure in patients with chronic ischaemic heart disease is often associated with a decrease in cardiomyocyte AgNOR numbers. There is also some evidence that cardiomyocyte AgNOR numbers are lower in cases with severe chronic ischaemic heart disease than in uncomplicated disease. ${ }^{2}$ On the basis of these findings, we assume that a significant decrease of interphase AgNORs in cardiomyocytes from cases with a severe course of chronic ischaemic heart disease might be provoked by an adaptive reaction of cardiomyocytes to diminished circulation at the level of pre-rRNA synthesis/ processing. A similar adaptative reaction of cardiomyocytes to ischaemia at the stage of cold cardioplegia seems to be of great value to the understanding of many aspects of prerRNA synthesis/processing regulation under different pathophysiological conditions and deserves further investigation.

Nucleolar organiser region silver staining in interphase nuclei is known to depend greatly on nucleolar proteins such as nucleolin, which plays an active role in both pre-rRNA synthesis and pre-rRNA processing. ${ }^{13}$ In addition to nucleolin, a family of small nucleolar RNAs (snRNAs) and fibrillarin are also involved in 
the post-transcriptional modification of human pre-rRNAs. ${ }^{14}$ In addition, there is some evidence that snRNA pairing with pre-rRNAs is related to ribose methylated pre-rRNA sites. ${ }^{14-16}$ Because the decrease of AgNOR numbers in cardiomyocytes at the stage of cardioplegia occurs at low temperature (18$20^{\circ} \mathrm{C}$ ), it might be concluded that the above pre-rRNA inactivation in cardiomyocytes cannot be a consequence of an enzymatic reaction, such as pre-rRNA ribose methylation. An explanation of this fast decrease/increase in AgNOR numbers in cardiomyocytes at the stages of cardioplegia and reperfusion, respectively, could be that there is a modification in the nucleolin/fibrillarin/pre-rRNA/snRNA transcription processing complex. The extent of the latter modification appears to be maximal at the stage of cold cardioplegia and total ischaemia. However, if this cardiomyocyte reaction to cardioplegia is less pronounced, cardiomyocyte damage will be provoked. To repair such hypothetical injury, non-protected cardiomyocytes react by increasing their activity, even at the stage of cold chemical cardioplegia. This is supported by our recent observation of a decrease in succinate dehydrogenase activity only in the group of patients with inadequate myocardial protection. ${ }^{1} \mathrm{Re}-$ garding the activation of protein synthesising mechanisms in myocardial fibroblasts at the stage of cold cardioplegia, it might be directly related to their reaction to various fibroblast activating cytokines, whose excess has already been demonstrated in cases with severe myocardial hypoxia. ${ }^{17}$ The exact nature of the cells that could be responsible for fibroblast growth stimulation in chronic ischaemic heart disease myocardium is unknown. Among potential candidates for this role are macrophages, lymphocytes, and damaged cardiomyocytes themselves. ${ }^{18}$ The fact that AgNOR numbers increase in fibroblasts from patients with chronic ischaemic heart disease might be an additional sensitive test of severe myocardial damage, including the cardioplegia stage. In conclusion, our data are in good agreement with those of Schaper et al, ${ }^{1920}$ who demonstrated that extensive cardiomyocyte degeneration and apoptosis in chronic ischaemic heart disease myocardium is coupled with active stimulation of extracellular matrix protein expression, resulting in a significant degree of reparative fibrosis. Overall, our findings show that the trend of AgNOR changes in surgical patients undergoing cardioplegia might be taken as a sensitive test for the functional state of the myocardium. Furthermore, it is note- worthy that the myocardium of patients with chronic ischaemic heart disease with antecedent myocardial infarction and severe heart failure is more vulnerable to ischaemia and needs special protection from total ischaemia. It is unclear what type of new cardioplegia technique should be used in these patients.

1 Mamaev NN, Kovalyeva OV, Amineva KhK, et al. Cardiomyocyte plastic function in surgical patients with ischemic heart disease as evaluated by nucleolar silver staining. Arkh Patol 1993;55:43-5. [In Russian.]

2 Mamaev NN, Gudkova AY, Amineva KK. AgNORs in the myocardium in ischemic heart disease complicated by heart failure: a postmortem study. $\mathrm{F}$ Clin Pathol: Mol Pathol 1998;51:102-4.

3 Hein S, Scholz D, Fujitani N, et al. Altered expression of titin and contractile proteins in failing human myocardium. titin and contractile proteins in failing
$7 \mathrm{Mol}$ Cell Cardiol 1994;26:1291-306.

4 Schaper J, Hein S, Scholz D, et al. Multifacetted morphological alterations are presented in the failing human heart. F Mol Cell Cardiol 1995;27:857-61

5 Hein S, Scheffold T, Schapper J. Ischemia induces early changes to cytoskeletal and contractile proteins in diseased human myocardium. I Thorac Cardiovasc Surg 1995;110: 89-98.

6 Schaper J, Scheld HH, Schmidt U, et al. Ultrastructural study comparing efficacy of five different methods of intraoperative myocardial protection in the human heart. $\mathcal{F}$ Thorac Cardiovasc Surg 1986;92:47-55.

7 Cankovic-Darracott S. Methods for assessing preservation techniques-invase methods (enzymatic, cytochemical). In: Engelman RM, Levitsky S, eds. A textbook of clinical cardioEngelman RM, Levitsky S, eds. A textbook of clinical cardio-
plegia. New York: Futura Publishing Company, 1982:43plegia.

8 Schaper W, Ito B, Buchwald A, et al. Molecular and ultrastructural basis of left ventricular reperfusion dysfunction. In: Kellermann JJ, ed. Advances in cardiology. Basel: $\mathrm{S}$ Karger, 1986:34-115.

9 Pichon H, Chocron S, Alwan K, et al. Crystalloid versus cold blood cardloplegia and cardiac troponin I release. Circulation 1997;96:316-20.

10 Mamaev NN, Gudkova AYa, Ainineva KhK, et al. The method of protein-synthesizing function assessment in human cardiomyocytes. Arkhive Anatomy Histology Embryology 1989;96:69-72.

11 Mamaev NN, Mamaeva SE. The structure and function of nucleolar organizer (NORs): molecular, cytological, and clinical aspects. Cytologiva 1992;34:3-25. [In Russian.]

12 Howell WM, Black DA. Controlled silver staining of nucleolus organizer regions with a protective colloidal developer: a one step method. Experientia 1980;36:101415

13 Serin G, Joseph G, Faucher C, et al. Localization of nucleoin binding sites on human and mouse preribosomal RNA. Biochimie 1996;78:530-8.

14 Cavaille J, Bachelleerie JP. Processing of fibrillarinassociated snoRNAs from pre-mRNA introns: an exonucleolytic process exclusively directed by the common stembox terminal structure. Biochimie 1996;78:443-56.

15 Cavaille J, Nicoloso M, Bachellerie J-P. Targeted ribosemethylation of RNA in vivo directed by tailored antisense methylation of RNA in vivo directed

16 Tollervey D. Small nucleolar RNAs guide ribosomal RNA methylation. Science 1996;273:1056-7.

17 Agocha A, Lee H-W, Eghbali-Webb M. Hypoxia regulates basal and induced DNA synthesis and collagen type 1 production in human cardiac fibroblasts: effects of transferring growth factor- $\beta 1$, thyroid hormone, angiotensin II and basic fibroblast growth factor. F Mol Cell Cardiol 1997;29: 2233-44.

18 Kelly R, Smitli TW. Cytokines and cardiac contractive function. Circulation 1997;95:778-81.

19 Schaper J, Speiser B. The extracellular matrix in the failing human heart. In: Hasenfuss G, Holubarsch Ch, Just H, et al, eds. Cellular molecular alterations in the failing human heart. Heidelberg: Steinkopff Verlag Darmstadt, 1992: 303-9.

20 Schaper J, Helii S, Elsasser A, et al. Degeneration, apoptosis and necrosis - the response to chronic heart disease of various causes [abstract]. F Mol Cell Cardiol 1996;28:A299. 